\title{
Structuration d'un espace migratoire. Le cas des émigrés albanais de Yougoslavie vers la Suisse
}

\begin{abstract}
Avant-propos
Cet article a été rédigé il y a une année. Il garde toute son actualité. Il est cependant indiqué d'y apporter quelques compléments d'information.

La situation économique et politique continue à se déteriorer au Kosovo. Le processus d'émigration se poursuit et d'aucuns, non sans raisons, parlent de "purification ethnique douce". Actuellement, les Albanais de Yougoslavie qui arrivent dans notre pays demandent l'asile politique ou vivent dans la clandestinité, le marché du travail helvétique leur étant fermé. Un nouveau groupe d'émigrés est constitué de jeunes réfractaires au service militaire.

En outre, l'émigration décrite dans l'article comme en premier lieu temporaire et masculine tend à devenir familiale et durable.

L'aggravation de la situation des droits de l'homme au Kosovo, le sort précaire des requérants d'asile kosovars dont la demande a été rejetée, émeuvent comme jamais auparavant certains milieux helvétiques (œuvres d'entraide, organisations de défense des droits de l'homme en particulier). La pression sur le Conseil fédéral s'est accrue pour que les requérants d'asile kosovars en instance de renvoi puissent rester au moins provisoirement en Suisse.

Dans ce contexte, l'émigration politique albanaise du Kosovo, très active dans notre pays, trouve plus facilement des appuis politiques extérieurs pour relayer ses revendications tout à la fois démocratiques et indépendantistes.

Il faut toutefois déplorer, parallèlement, que l'image des Albanais du Kosovo est ternie pour un certain nombre de ressortissants de cette région s'adonnant au trafic de drogue et d'armes. II reste à espérer que ces comportements délictueux n'alimentent pas dans une trop forte mesure la xénophobie à l'égard de certaines catégories d'étrangers, les émigrés albanais de Yougoslavie en particulier.
\end{abstract}

\section{Introduction}

Ce n'est qu'à partir du milieu des années septante que la sociologie des migrations abandonne progressivement ses concepts d'intégration et d'acculturation où le parcours du migrant est interprété selon le modèle du continuum, processus linéaire de modernisation dont l'aboutissement est l'assimilation. En précurseurs, BÜCHLER \& BÜCHLER (1975) analysent l'émigration de Galiciens vers la Suisse en tenant compte de l'importance de la recréation constante de liens sociaux dans l'espace migratoire Suisse-Galice, forgeant et maintenant la conscience et l'identité collective du groupe.

Dès le début des années huitante, l'expérience migrante se conçoit aussi comme celle d'une alternative à la contrainte de l'assimilation/intégration et du retour au pays de départ (DE CERTEAU: 1985). En Suisse, la reconnaissance d'un espace migratoire structuré pour de nombreux groupes d'é(im)migrés tant par les références à la région d'origine qu'à celles du pays d'expatriation transparait dans les travaux de GIORDANO (1984), d'HETLAGE \& HETLAGE (1984). Le premier examine les processus de réacculturation et de différenciation culturelle des migrants suite à leurs déplacements réguliers entre la région d'origine et la société d'accueil. Les seconds forgent le concept d'entre-deux-models culturel.

L'objectif de cette contribution est, en se basant sur l'étude d'un groupe à très forte solidarité et cohésion ethnique (les émigrés albanais de Yougoslavie), de corroborer d'une part la validité du concept d'espace migratoire. D'autre part, il s'agit de montrer que l'espace migratoire des Albanais de Yougoslavie est fortement polarisé par les références à la société d'origine, ce qui nuit à leur intégration dans le pays d'emploi. Pour le démontrer, nous présenterons (ce qui rend inévitable un certain schématisme) l'espace migratoire des Albanais de Yougoslavie à la fois comme un espace de reproduction socio-économique et identitaire.

\section{La polarisation de l'espace migratoire}

L'espace migratoire peut être défini comme le support d'un entre-deux, pratiqué, structuré et perçu symboliquement par les références au pays d'origine et d'immigration (DE RUDDER \& TABOADA-LEONETTI: 1982, 44-45). Raisonner en termes d'espace migratoire nous parait d'autant plus pertinent dans le cas d'une émigration récente. Or l'émigration des Albanais de Yougoslavie vers la Suisse, bien qu'elle remonte au début des années septante, ne se fait quantitativement importante qu'à partir du début des années huitante. C'est pour l'essentiel un phénomène spontané qui se met en place par le biais des relations et connexions familiales. L'émigration vers la Suisse à partir du Kosovo est en quelque sorte une alternative aux flux migratoires de la fin des années soixante vers l'Allemagne fédérale stoppés au milieu des années septante.

Denis Torche, Fontany 3, 1470 Estavayer-le-Lac. 
Il est impossible de chiffrer avec exactitude le nombre d'émigrés albanais de Yougoslavie dans notre pays puisque l'Office fédéral des étrangers recense seulement des "Yougoslaves», indépendamment de leur appartenance ethnique ou nationale. Néanmoins, on peut procéder à des estimations selon divers recoupements obtenus auprès de sources consulaires, syndicales et des intéressés eux-mêmes. Il y avait grosso-modo vingt mille émigrés albanais de Yougoslavie en Suisse au début des années huitante, quarante mille en 1990, chiffre auquel il faut ajouter plusieurs milliers de personnes en situation irrégulière.

Ce qui frappe, c'est qu'il s'agit d'une émigration conservant un caractère temporaire accentué. C'est donc, en dépit d'une certaine stabilisation par le biais des regroupements familiaux, aussi une émigration à forte précarité d'un point de vue statutaire, caractérisée par un nombre important de saisonniers, de requérants d'asile, de travailleurs clandestins ou semi-clandestins.

Toutefois, vouloir expliquer par le caractere récent et précaire de la migration la forte polarisation par les références à la société d'origine de l'espace migratoire des Albanais de Yougoslavie ne suffit pas. Sans doute le statut de saisonnier retarde l'intégration dans le pays hôte et peut même indirectement contribuer, par la mobilité accrue qu'il induit, à la vitalité des réseaux de communication dans le cadre notamment de la mobilisation politique et nationaliste des émigrés (TORCHE: 1989, 130).

Les enquêtes de terrain que j'ai menées auprès d'immigrés bénéficant d'un permis d'établissement, en théorie bien intégrés dans le pays d'emploi, montrent que chez eux aussi les références au pôle de la région d'origine demeurent prédominantes. Comment l'expliquer? Nous montrerons d'une part que dans le cadre de la reproduction socio-économique de l'espace migratoire la structure familiale d'origine induit des stratégies d'adaptation économique tournées vers la région de départ. D’autre part, l'espace de reproduction identitaire est caractérisé par la vigueur du mythe du retour. Parce que le Kosovo est le lieu de cristallisation de deux nationalismes concurrents (serbe et albanais), les émigrés perçoivent la migration comme celle d'une situation de mobilité contrainte.

\subsection{Reproduction socio-économique de l'espace migratoire}

L'émigration des Albanais de Yougoslavie vers la Suisse présente un caractère de continuité avec d'anciens mouvements migratoires à caractère saisonnier, masculin et balkanique du sud-est de la Serbie, la petchalba. Dans l'ex-Yougoslavie, les mouvements migratoires des Albanais vers les républiques du nord plus prospères (Slovénie et Croatie) sont le plus souvent le fait d'hommes (maçons, artisans, boulangers) ayant laissé temporairement leur famille dans la région d'origine. Les Albanais de Macédoine, nombreux à tenir des cafés et des pâtisseries sur la côte dalmate, gardent des liens familiaux très vifs avec leurs villages d'origine.
Tenus longtemps à l'écart du développement économique, social et culturel, les Albanais de Yougoslavie sont restés plus ruraux et jusqu'au début des années huitante moins mobiles que les autres populations de Yougoslavie. Cette mobilité tardive et perçue comme temporaire est à corréler avec une organisation familiale patriarcale de type clanique et à l'enracinement paysan.

A l'absence de traditions fortes dans la mobilité de la population albanaise du Kosovo, il faut ajouter un manque d'information sur l'embauche et l'encadrement à l'étranger; la création tardive (en 1968) d'un Service régional de l'emploi pour l'étranger, au moment où ailleurs en Yougoslavie les départs pour l'étranger étaient déjà massifs. Néanmoins, diverses estimations laissent penser que près de deux cent mille Albanais de Yougoslavie se sont entre 1970 et 1990 expatriés vers divers pays d'Europe occidentale: cent mille vers l'Allemagne fédérale, soixante mille vers la Suisse (y compris les travailleurs temporaires de tous statuts), quarante mille vers la Belgique, la France, l'Autriche, la Scandinavie.

Par rapport à d'autres groupes d'émigrés en provenance du bassin méditerranéen, l'émigration des Albanais de Yougoslavie vers la Suisse présente une plus forte propension à être masculine et temporaire. Il est intéressant de noter que selon le recensement de 1981, des 19850 organisations domestiques du Kosovo ayant des membres travaillant à l'étranger, 16692 d'entre elles n'avaient qu'un travailleur migrant; 2625, deux membres à l'étranger; 533 , trois et plus de membres. Il en résulte que la discontinuité entre région d'origine et pays d'immigration est plus apparente que réelle dans de nombreux cas, les émigrés demeurant dépendants des stratégies d'adaptation socio-économiques de familles généralement étendues.

Le système de parenté des Albanais de Yougoslavie est de type patriarcal. Le terme de famille y est d'emploi récent. Les Albanais emploient le mot shpie pour désigner toutà-la-fois maison et organisation domestique. Aujourd'hui encore, les Albanais de Yougoslavie se regroupent, surtout en milieu rural, en organisations domestiques, basées sur l'extension patri- et fratrilinéaire, formant des unités résidentielles et économiques très soudées. Bien que les plus grandes organisations domestiques albanaises (appelées zadruga chez les Slaves du Sud) soient sur le déclin, s'étant adaptées aux processus de modernisation, il n'en demeure pas moins que les organisations domestiques présentent toujours un nombre élevé de membres. La taille moyenne des ménages a même augmenté au Kosovo entre 1948 et 1981 (de 6,3 à 6,9 membres) à l'inverse du reste du pays (de 4,3 à 3,8 membres). En 1961, le Kosovo comptait 43707 organisations domestiques de plus de huit membres $(28,7 \%$ de la population); en 1981, $80823(35,3 \%)$.

La structure familiale d'origine nous paraît donc être un facteur qui explique que dans maints cas les stratégies socio-économiques des émigrés sont fortement orientées vers la région d'origine. Nous allons l'illustrer par un exemple concret en analysant l'adaptation socio-économique d'une organisation domestique étendue, ayant des 
membres travaillant à l'étranger. Cet exemple ne prétend pas à l'exhaustivité mais est néanmoins représentatif d'une partie des organisations domestiques rurales du Kosovo pour lesquelles la migration est devenue le vecteur principal de l'adaptation économique, voire même de la survie matérielle.

Il s'agit d'une organisation domestique regroupant sous le même toit trois frères, leurs épouses et leur descendance, soit dix-huit personnes. En 1989, seul le second frère travaillait en Suisse. L'émigration fut rendue nécessaire par le fait que la viabilité de l'organisation domestique ne put être assurée par le domaine de 2 ha et l'élevage, placés sous la responsabilité du frère aîné, et le gain du frère cadet, comptable dans un hôtel.

En raison des faibles revenus tirés du domaine - estimés à 100 Frs. par mois en 1989 - et du travail salarié du frère cadet (150 Frs. par mois), l'adaptation économique de l'organisation domestique repose surtout sur les économies du frère émigré. Ce dernier envoie l'équivalent de 500 Frs. par mois. En 1990, le fils aîné a rejoint en Suisse le second frère. Au moment de l'enquête, il venait de trouver un emploi clandestin.

Le frère aîné gère et redistribue l'argent aux membres de la "famille étendue». Le solde est investi dans l'achat de matériel de construction ou agricole. Une petite partie des économies est investie à des fins de reproduction sociale: fêtes, invitations et parfois coup de pouce aux familles les plus pauvres du village, ce qui confere l'honneur familial vis-à-vis d'autrui.

Le frère émigré ne peut donc faire fructifier ses économies comme il l'entendrait puisqu'elles sont assujetties à la reproduction socio-économique de l'organisation domestique. La précarité de l'adaptation économique de l'organisation domestique, reposant sur les gains de l'étranger, empêche l'épouse et les quatre enfants du frère émigré de revenir rejoindre ce dernier de manière durable en Suisse. En 1990, le frère émigré vivait en Suisse depuis dix ans. L'un des buts de l'émigration est de bâtir deux nouvelles habitations afin que les couples puissent vivre de manière plus indépendante. Mais seule une vaste chambre d'hôtes a pu être réalisée. Il s'agit d'une grande pièce réservée aux hommes que l'on appelle oda e burrave (chambre des hommes), ce qui traduit bien le maintien des normes patriarcales de la sociéte albanaise. Un autre projet s'est toutefois concrétisé: l'ouverture d'une petite épicerie qui permet de diversifier l'économie de l'organisation domestique.

Pour confirmer la force de l'utopie de retour et la perpétuation des mécanismes d'affiliation ethnique, analysons la situation d'émigrés ayant procédé au regroupement familial. On constatera alors que les formes de cooperation économique à destination de la région d'origine se poursuivent entre frères, vivant pourtant séparément avec leur famille restreinte (l'épouse et les enfants) en situation migratoire.

Dans la cité fribourgeoise d'Estavayer-le-Lac, les Albanais de Yougoslavie, detenteurs de permis $\mathrm{B}$ ou $\mathrm{C}$, ont pour la plupart procédé au regroupement familial. Ils considèrent néanmoins leur séjour dans le pays d'emploi comme provisoire. Le bref aperçu des stratégies socioéconomiques de l'une de ces familles migrantes le démontre. M. M. est détenteur d'un permis C. Il vit à Estavayer-le-Lac avec son épouse et trois enfants en bas âge. Deux de ses frères vivent aussi dans la même cité; l'un, qui a un permis B, a été rejoint par sa femme et ses quatre enfants (déjả adolescents) en 1991, l'autre, saisonnier, a laissé sa famille dans le village d'origine.

Entre les trois frères la cooperation économique à destination de la région d'origine se poursuit. La contribution de chacun n'est pas évaluée en termes monétaires puisqu'ils se considerent - bien que vivant en immigration comme appartenant toujours à la même organisation domestique. Les économies des trois frères (très inégales en raison de séjours de durée différente en Suisse) sont toujours mises en commun.

Elles ont servi à acheter deux parcelles de terre agricole, l'une de 2 ha et demi, l'autre de 1 ha. Ces parcelles sont cultivées par un paysan auquel les frères lui laissent le $50 \%$ du produit de la terre. Une autre parcelle d'un ha et demi a été achetée pour bâtir les futures maisons des frères émigrés. Lorsque l'on procédera au partage des biens fonciers, les frères hériteront de parts égales, indépendamment de la contribution monétaire de chacun.

\subsection{Espace de reproduction identitaire, intégration et mythe du retour}

L’intégration des étrangers peut être définie comme la possibilité des interactions multiples avec la société d'accueil, par exemple l'insertion sociale, la promotion professionnelle, sans pour autant que soit exigée la conformité à nos us et coutumes. Selon cette définition il est incontestable que l'intégration des Albanais de Yougoslavie est très partielle, voire même peu souhaitée par les émigrés eux-mêmes. Néanmoins, le cas des émigrés albanais de Yougoslavie nous permet de relativiser la place centrale qui fut longtemps accordée à la dimension culturelle de l'intégration dans la sociologie des migrations, occultant les aspects économiques, sociaux, politiques de l'insertion (DE RUDDER: 1985, 24).

Certes, le recensement de 1981 nous apprend que 48,4\% des émigrés du Kosovo étaient des agriculteurs. Et il est vrai que nombre d'entre eux sont faiblement scolarisés, ce qui leur pose de sérieux problèmes d'adaptation socioculturelle dans le pays d'expatriation. Néanmoins, le relevé de la formation socio-professionnelle d'origine des émigrés albanais de Yougoslavie employés à Estavayerle-Lac montre que tous ont achevé leur scolarité secondaire. Pourtant aucun d'entre eux n'a réussi à ce jour à s'engager dans un processus de mobilité verticale. Tous déclarent considerer leur sejour en Suisse comme provisoire et limitent fortement leurs interactions avec leur environnement immédiat.

Ce «refus» de l'intégration a été explicité par la structure familiale d'origine et la force des stratégies socio-économiques des émigrés dirigées vers la région d'origine. A ce- 
la il faut ajouter que les Albanais de Yougoslavie sont engagés dans un vigoureux processus d'affirmation nationale dont les effets ne manquent pas de se répercuter en situation migratoire: radicalisation du sentiment national avec une mobilisation collective sur une base ethnique, survalorisation des références communautaires servant de palliatif au manque de sentiment d'appartenance au nouvel environnement, etc.

L'ètude de l'espace de reproduction identitaire des émigrés albanais de Yougoslavie pouvant faire l'objet d'une thèse, nous nous contenterons ici d'en cerner l'un des aspects: le discours en vigueur sur les causes de la migration.

Dans ce qui est l'ex-Yougoslavie, c'est au Kosovo que se pose de manière la plus cruciale le problème de l'emploi. Selon le recensement de 1981, dans cette province du sud de la Serbie à forte majorité albanaise $(77,5 \%$ d'Albanais en 1981) sur une population de 1500000 personnes, seules 178000 d'entre elles étaient employées. A partir des années huitante, la population en âge de travailler croît de plus de 3\% par an. L'écart du taux d'activité du Kosovo augmente donc avec le reste de l'ex-Yougoslavie, oú pour la même période, le nombre de personnes en âge de travailler croît en moyenne de $0,9 \%$ par an.

Il n'en demeure pas moins que tant les Serbes que les Albanais de Yougoslavie perçoivent l'émigration de leurs compatriotes comme contrainte. Les premiers qui representaient $27,4 \%$ de la population du Kosovo en 1961 ne comptent que plus que pour $14 \%$ en 1981. Cette diminution est relative puisqu'elle n'est pas due seulement à l'émigration mais aussi au taux d'accroissement naturel plus élevé de la population albanaise. Quoi qu il en soit, le thème de l'émigration des Serbes a été présenté par les autorités nationales-communistes de Belgrade comme la conséquence des "attitudes génocidaires de la population albanaise afin de créer un Kosovo ethniquement pur». S’il est vrai que dans certains cas les Albanais ont fait pression sur les Serbes en leur rachetant leurs terres (payées souvent en devises par les émigrés), l'émigration des Serbes s'explique par un sentiment général d'insécurité et par l'absence de perspectives économiques.

De leur côté, les Albanais de l'émigration avançaient déjà la thèse du colonialisme interne alors que le Kosovo jouissait d'un large statut d'autonomie. Cette these fut facile à diffuser d'autant plus que le sous-sol du Kosovo est très riche en minerais. En m'entretenant avec les émigrés albanais de Yougoslavie, j'ai pu constater que mes interlocuteurs étaient pour la plupart persuadés qu'ils pourraient rentrer et travailler dans leur province d'origine pour autant que le Kosovo obtienne le statut de république. Il est révélateur que lors des innombrables manifestations d'Albanais de Yougoslavie à l'étranger réclamant le statut de république pour leur province, apparaisse frequemment l'exigence du retour des travailleurs migrants dans leur région d'origine.

Si au cours des années huitante, la thèse du colonialisme interne permettait d'occulter les responsabilités locales dans le mal-développement du Kosovo (la planification économique était de la compétence des autorités albanaises de Pristina), aujourd'hui les griefs de la population albanaise sont fondés: avec la suppression progressive du statut d'autonomie du Kosovo à partir de 1989, conduisant, entre autres, à une situation de discrimination économique. Des dizaines de milliers d'Albanais ont perdu leur emploi pour des raisons politiques. Ils ont été remplacés par des Serbes, notamment dans les postes à responsabilité.

Niant l'évidence, préoccupées par l'albanisation croissante du Kosovo, les autorites socialistes serbes (tout comme l'opposition nationaliste d'ailleurs) mettent une condition préalable à tout règlement du problème du Kosovo: rétablir l'équilibre ethnique dans la province en inversant les flux migratoires. Les Albanais y voient par conséquent la menace d'une nouvelle vague de colonisation serbe. D'ou le paradoxe d'une région déjà frappée par un chômage endémique mais où l'exacerbation nationaliste des uns et des autres les encourage à s'enraciner sur place.

La perception des Albanais est bien résumée dans un article du périodique en langue albanaise de la culture, des arts et des lettres du Kosovo (Fjala, no 25, p. 2, 1 novembre 1990). On peut y lire que: "Le pouvoir militaro serboslave cherche à imposer au peuple albanais l'émigration vers l'étranger... C'est un devoir d'expliquer aux jeunes les conséquences de la migration, qui sont fatales pour les familles et notre peuple. Chacun doit donner sa contribution là où il se trouve: dans l'éducation de la jeunesse, à l'école, à la faculté, au sein de l'entreprise, de la famille, etc., car il serait terrible d'avoir demain des entreprises sans jeunes, des maisons qui ne résonnent plus de la voix des enfants, des amphithéâtres sans étudiants.. Nous lançons un appel aux parents, aux. frères, aux amis, aux enseignants et à tous ceux qui en ont la possibilité afin d'empêcher l'émigration ethnique de nos frères, de nos jeunes, de notre futur:"

Devant l'aggravation des discriminations de tous ordres a l'encontre de la population albanaise du Kosovo, les émigrés sont appelés à jouer un rôle croissant dans les stratégies de résistance. Il en résulte un resserrement des liens intra-ethniques et une pression sociale et morale du groupe sur l'individu de plus en plus forte. Cette pression communautaire non seulement fait office de rempart à l'assimilation mais rend plus problematique tout projet d'intégration dans la société d'accueil. Les obligations des émigrés ne consistent plus seulement en apports financiers à destination des organisations domestiques dont ils font partie. Il faut aussi aider financierement par des versements à des fonds de solidarité un nombre croissant de familles du Kosovo, dénuées de toutes ressources. C'est le cas de centaines de familles de la région miniere de Mitrovica qui ne peuvent compter sur l'apport de l'agriculture, les retombées de zinc rendant les terres improductives. Les émigrés sont aussi encouragés à verser $3 \%$ de leur salaire au gouvernement en exil du Kosovo.

La force des identifications de référence en situation migratoire (manifestations autonomistes, recréation symbolique de l'identité nationale par des chansons histori- 
ques ou la commémoration d'anniversaires historiques) pallie et occulte en quelque sorte les difficultés sociales et matérielles que rencontrent les émigrés. Il est frappant de constater que les émigrés albanais de Yougoslavie ne formulent aucune revendication relative à leur statut en Suisse (les associations italiennes et espagnoles avaient revendiqué une meilleure intégration par le biais des droits politiques par exemple).

Les émigrés albanais de Yougoslavie luttent eux pour que l'opinion helvétique les soutienne dans leurs revendications nationales et démocratiques. Ils cherchent aussi à ce que leur particularisme culturel soit mieux reconnu en Suisse. Par exemple dans le domaine linguistique avec l'exigence de textes les concernant rédigés non pas en serbo-croate mais en langue albanaise (la Commission fédérale pour le problème des étrangers a depuis peu rédigé aussi en langue albanaise son dépliant à l'intention des saisonniers). De même dans le domaine syndical, les Albanais se mettent à revendiquer des informations dans leur langue mais aussi que leur ethnie soit mieux représentée dans les postes de délégués syndicaux d'origine étrangère.

\section{Conclusion: en termes de reformulation culturelle}

Bien qu'ayant insisté ici sur le pôle d'origine de l'espace migratoire, il ne saurait être question de nier l'apport des influences de la société d'accueil. Il s'agit toutefois moins d'un processus d'acculturation de valeurs spécifiques à la société d'accueil que de celles plus générales de la société occidentale: avec l'importance mise dans la consommation et la modernisation. C'est ainsi que l'espace de reproduction socio-économique inclut des innovations et des signes de prestige que les émigrés se sont appropriés et qu'ils transmettent avec plus ou moins de réussite dans la société d'origine.

Mais plutôt que de réfléchir en termes d'acculturation, nous pouvons proposer le terme de reformulation culturelle pour ce qui concerne la première génération d'émigrés tout au moins. En effet les émigrés assimilent les innovations (sur le plan technique) et les influences culturelles en les réincorporant selon leur propre systeme de valeur. Par exemple presque tous les émigrés que j'ai fréquentés (même récemment arrivés dans notre pays) disposaient d'un magnétoscope. Mais celui-ci sert dans la plupart des cas a diffuser des programmes (films, folklore, actualité, etc.) en rapport avec la société d'origine. Un autre exemple est celui de l'alimentation, un domaine, comme on le sait, de forte résistance culturelle. Avant leur arrivée en Suisse, de nombreux saisonniers n'avaient jamais mangé de spaghettis. Mais ils les préparent en les réincorporant dans leur imaginaire alimentaire: avec une sauce à base de yoghurt et d'ail.

Enfin, la prise en compte des deux pôles de l'espace migratoire nous permet de démontrer que la culture des émigrés albanais de Yougoslavie est sujette à de constantes redéfinitions. La migration des Albanais de Yougoslavie est caractérisée par un va-et-vient constant de membres apparentés. Cette mobilité due à la structure familiale d'origine, aux conditions économiques mais aussi politiques prévalant dans la région de départ permet de découvrir un aspect peu étudié dans la sociologie des migrations: la redéfinition "situationnelle" des comportements socio-culturels marquée par des périodes successives de renforcement et de relâchement du contrôle social dans l'espace migratoire. Il arrive en effet que le relâchement des normes sociales et morales en immigration se voit soudainement interrompu sous l'effet de l'arrivée de membres apparentés, détenteurs d'autorités.

Un exemple révélateur est celui de la prescription des comportements féminins par les hommes. En immigration, les traits patriarcaux de la société d'origine tendent à s'atténuer notamment dans les domaines de l'habillement féminin et de la ségrégation socio-spatiale en fonction du sexe. Mais il suffit de la présence d'un pere, d'un oncle, voire d'une connaissance du village d'origine pour que d'anciens comportements prescrits par la coutume soient à nouveau en vigueur. Ainsi l'épouse de X. M. qui avait pris l'habitude de revêtir des pantalons et qui, lors de la visite de son pere, les abandonna au profit de longues robes. Autre exemple: l'épouse de M. I., qui lors d'une visite à son mari de compatriotes originaires du même village d'origine, resta confinée dans la cuisine de l'appartement, le salon retrouvant une fonction analogue à celle de la chambre des hommes dans le village d'origine. L'épouse de M. I. ne fit qu'une brève apparition pour saluer les convives et apporter le repas.

En définitive, si les émigrés réussissent à maintenir un certain nombre de traits culturels (bien quappauvris et parfois réinventés en raison de l'éloignement des bases référentielles et d'une nécessaire adaptation à l'espace migratoire), ce n'est pas parce que ces traits résisteraient d'eux-mêmes au nouvel environnement mais bien parce qu'ils découlent d'une volonté consciente de maintenir l'identité-appartenance. Plus que chez d'autres groupes d'immigrés, l'exemple des Albanais de Yougoslavie indique que les perspectives de l'intégration et de l'acculturation sont le plus souvent une issue solitaire: parce qu'il s'agit d'une émigration qui s'est mise en place dans le cadre de tres solides liens familiaux et qui, de plus, en raison d'un très vigoureux sentiment d'affirmation ethnico-nationale, tend à exclure l'individu qui ne se conformerait pas aux regles sociales et morales prescrites par le groupe.

Il en résulte qu'une double allégeance à la fois au groupe et à de nouvelles valeurs est rendue très difficile. Toutefois on constate que même chez les individus qui sont sortis du groupe de référence, l'intégration ne signifie pas assimilation. La conscience de l'identité nationale d'origine demeure très forte, bien qu'elle n'entraîne plus forcément de participation aux activités de recréation communautaire et de revendications à caractere national. 


\section{Résumé:}

Cette contribution analyse la structuration de l'espace migratoire des Albanais de Yougoslavie ayant émigré vers la Suisse. Elle cherche à démontrer qu'il s'agit d'un espace fortement polarisé par les références à la société d'origine. Dans le cadre de la reproduction socio-économique de cet espace, nous montrons que la structure familiale d'origine de type patriarcal oriente fortement les stratégies socio-économiques des émigrés vers la région de départ. De plus, le conflit de nationalités qui oppose Albanais et Serbes au Kosovo dénature les causes réelles de l'émigration, les émigrés percevant cette dernière comme une situation de mobilité contrainte. C'est ainsi que les conditions tant socio-économiques qu'identitaires qui prévalent dans la région d'origine tendent à favoriser le maintien chez les émigrés d'un fort sentiment d'identité-appartenance et de loyauté au groupe. Cela nuit à leur intégration tout comme à leur mobilité verticale dans le pays d'expatriation.

Ayant insisté sur le pôle d'origine de l'espace migratoire, nous nous interrogeons aussi à la fin de cet article sur les questions d'acculturation que la société d'accueil peut provoquer chez les émigrés. En ce qui concerne la première génération d'émigrés, nous croyons dans notre cas qu'il serait plus juste de parler de reformulation culturelle. Nous tentons de le démontrer en montrant que les émigrés, s'ils assimilent les innovations (sur le plan technique) et de nouvelles influences culturelles, les réincorporent selon leur propre système de valeur.

\section{Bibliographie:}

BACKER, B. (1979): Behind the Stone Walls. Changing Household Organization among the Albanian in Yugoslavia, Thèse, Oslo.
BÜCHLER, H. C., \& BÜCHLER, J. M. (1975): Los Suizos: Galician Migration to Switzerland, in SAFA, H. I., \& DU TOIT, B. M. (Ed.), Migration and Development. Implications for Ethnic Identity and Political Conflict, pp.17-29, Mouton, The Hague, Paris.

CVIJIC, J. (1918): La péninsule balkanique: géographie humaine, Armand Colin, Paris.

DE CERTEAU, M. (1985): L'actif et le passif des appartenances. Dans: Esprit no 6, pp.155-171.

DE RUDDER, V. (1985): L'obstacle culturel: la différence et la distance. Dans: L'homme et la société no 77/78, pp. 23-49.

DE RUDDER, V., \& TABOADA-LEONETTI, I. (1982): La cohabitation pluriethnique: espace collectif, phénomènes migratoires et relations sociales. Dans: Pluriel no 31, pp. 37-54.

GIORDANO, C. (1984): Zwischen Mirabella und Sindelfingen. Zur Verflechtung von Uniformierungs- und Differenzierungs-Prozessen bei Migrations-Phänomenen. In: Schweizerische Zeitschrift für Soziologie Nr. 2, S. 437-464.

HETLAGE, A. \& R. (1984): Kulturelle Zwischenwelten. Fremdarbeiter - eine Ethnie? In: Schweizerische Zeitschrift für Soziologie Nr. 2, S. 357-404.

ISLAMI, H. (1979): Le développement démographique du Kosovo. Dans: Revue géographique de l'est no 1/2, pp. 87-100.

ISLAMI, H. (1987): Problème de la natalité chez les Albanais. Dans: Recherches albanologiques no 4, pp. 249-270.

POINARD, M., \&ROUX, M. (1977): L'émigration contre le développement: le cas portugais et yougoslave. Dans: Tiersmonde no 69, pp. 21-53.

ROUX, M. (1982): Le Kosovo: développement régional et intégration nationale en Yougoslavie. Dans: Hérodote no 25, pp. 10-48.

ROUX, M. (1990): Minorité nationale, territoire et développement: les Albanais en Yougoslavie, Thèse, Toulouse.

TORCHE, D. (1989): Les liens entre l'identité nationale et le fait migratoire: le cas des Albanais de Yougoslavie. Dans: Revue suisse de sociologie no 1, pp.115-138. 